\title{
RARE ASSOCIATION OF POLYNEUROPATHY AND CROHN'S DISEASE: A CLINICOPATHOLOGICAL STUDY OF 4 CASES
}

\author{
Evangelia Kararizou, Panagiota Davaki, Konstantinos Speggos, Elevtherios Stamboulis
}

Section of Neuropathology, Neurological Clinic of the University of Athens, Aeginition Hospital, Athens, Greece

\begin{abstract}
The purpose of this study is to investigate the clinical, electrophysiological and pathological features of neuropathy in patients with Crohn's disease.

Biopsies were selected from over 700 sural nerve biopsies. The diagnosis of Crohn's disease was based on established clinicopathological criteria. Complete laboratory, clinical, electrophysiological and pathological studies were performed in all cases.

Nerve biopsies of 4 patients were diagnosed as neuropathy and Crohn's disease. Distal symmetrical sensorimotor polyneuropathy was the pattern of neuropathy. The pathological features were mixed, demyelination with predominant axonal degeneration and a varying pattern of myelinated fiber loss. There were no vasculitic changes found.

We conclude that patients with Crohn's disease are complicated frequently with polyneuropathy, and as remission depends on immunosuppressive therapy, it is important to recognise it in the early stage. The diagnosis of polyneuropathy is based on clinical and electrophysiological studies, but precise histology, immunohistochemistry and morphometric studies of the peripheral nerve biopsy may be decisive in establishing the diagnosis.
\end{abstract}

Key words: Crohn's disease, polyneuropathy, axonal degeneration, demyelination.

\section{Introduction}

Crohn's disease (CD) is a chronic inflammatory disease involving the gastrointestinal tract. The aetiology is unknown. Patients usually present with abdominal pain and diarrhoea, frequently accompanied by fever and weight loss. Extraintestinal complications of CD are common and include mainly arthritis, ocular, dermatologic manifestations and neurological abnormalities [1-4]. Peripheral neuropathy $(\mathrm{PN})$ is one of the most frequent reported neurological complications in patients with $\mathrm{CD}$ [5-10]. As the peripheral nerve disease often dominates the clinical picture, the peripheral nerve biopsy may be very useful to establish the diagnosis. In fact, a few cases of CD with examination of the peripheral nerve biopsy have been published $[11,12]$.

The present retrospective study examines the clinical, electrophysiological, histopathological and mor- phometric features of 4 patients with CD in order to promote the early diagnosis of the neuropathy. Patients with $\mathrm{CD}$ are complicated frequently with polyneuropathy and it is important to recognise it, because the course of disease depends on immunosuppressive therapy introduced in the early stage.

\section{Material and methods}

\section{Patient selection and clinical data}

Biopsy specimens were selected from over 700 sural nerve biopsies performed at the Section of Neurology, Neurological Clinic of Athens University Hospital, during the last 20 years. The selection of patients was done according to the criteria for the diagnosis of CD [13]. Biopsies from patients with other causes of peripheral neuropathy such as malignancy, diabetes, vitamin $\mathrm{B}_{12}$ deficiency and metronidazole treatment 
were excluded. All patients were referred to us for investigation of the polyneuropathy. Clinical data of the patients were obtained retrospectively from medical files. All patients had endoscopic, colonoscopic examination with biopsy, and a CT scan of the abdomen examinations done. Clinical severity classification was used according to the European Crohn's and Colitis Organisation (ECCO) [14]. Routine laboratory tests were performed in all cases at the time of diagnosis. Neurological interviews and examinations have been carried out in all patients prior to nerve biopsy by at least one neurologist. Muscle strength testing was scored in the 5 point Medical Research Council scale. The patients' functional state was estimated using the modified Rankin scale. Complete serological tests including CRP, $\mathrm{C}_{3}, \mathrm{C}_{4}$, ANA, ANCA, anti-DNA, RF, cryoglobulin, immunoelectrophoresis have been obtained in all cases. Serum and urine specimens were tested for monoclonal protein. CSF was investigated in all cases. Nerve conduction studies and electromyography (EMG) were performed in each patient at the EMG laboratory of our institution.

Neuropathy type was classified as mononeuritis multiplex, distal symmetrical sensorimotor polyneuropathy, or asymmetrical/overlapping neuropathy [15].

\section{Histological techniques}

Biopsy of the whole sural nerve was performed under local anaesthesia $[16,17]$. Specimens were divided into three to five sections, each about $1 \mathrm{~cm}$ in length. One piece was fixed in $10 \%$ formaldehyde embedded in paraffin and cut transversely and longitudinally in sections of $7 \mu \mathrm{m}$ thickness. The sections were stained with haematoxylin and eosin (HE). Another piece was fixed in Flemming's solution for $24 \mathrm{~h}$, dehydrated in alcohol, embedded in paraffin wax and cut transversely and longitudinally in serial sections of $7 \mu \mathrm{m}$ thickness. The sections were stained with Weigert Pall. A third piece was fixed in $1 \%$ glutaraldehyde, stained for $24 \mathrm{~h}$ in $1 \%$ osmium tetroxide, macerated in glycerol and then teased apart under a dissecting microscope in order to isolate single nerve fibers. At least 50 fibers were sampled and assessed for pathological conditions based on the criteria of Dyck et al. [18]. The specimens for semithin sections and electron microscopy were fixed in a solution of $2.5 \%$ glutaraldehyde in Sorenson buffer and embedded in epoxy resin. Semithin sections were stained with toluidine blue. Ultrathin sections were stained with uranyl acetate and lead extract and examined with a Philips EM 201 electron microscope. Another portion of the nerve was prepared for immunohistochemical staining. Immunoperoxidase procedures were used for polyclonal antibodies $\operatorname{IgG}, \operatorname{IgM}, \operatorname{Ig} A, C_{3}$.

Pathologic changes were diagnosed and classified as predominantly axonal, demyelinating or mixed axonal and demyelinating based on both teased fibers and resin sections, according to established criteria [19].

\section{Morphometry}

Morphometric analysis of myelinated fibers was performed using VIDS III and OPTOMAX V image analysis system, connected with a microscope by a colour camera and included the measuring of the fascicle area, number, density and mean diameter of the myelinated fibers and fiber-size distribution histograms, myelin sheath thickness, mean axon diameter and g ratio (diameter of an axon without its myelin to the diameter of the axon with its myelin).

\section{Results}

\section{Clinical features}

Seven cases fulfilled the criteria for CD [13]. Three of them were excluded because there were other causes of peripheral neuropathy (one had a diagnosis of diabetes and vitamin $\mathrm{B}_{12}$ deficiency, one had vita$\min B_{12}$ deficiency and one was treated with metronidazole). The final number of patients included in the study was four, 3 female and one man.

Abdominal pain, diarrhoea, anaemia, fever (37.2$37.5^{\circ} \mathrm{C}$ ) and weight loss ( $>5 \%$ body weight) were the most frequent clinical manifestations from the history of patients. There were no other extraintestinal symptoms or complications (obstruction, fistulae, or abscesses). There were no arthralgias or myalgias. Neuropathy symptoms began $10.3 \pm 2$ years after $\mathrm{CD}$ onset. Neurological symptoms were mainly complaints in all patients during hospitalization in our department and all symptoms of $\mathrm{CD}$ were under control at presentation (except for episodes of mild diarrhoea in one of them). Routine haematological and biochemical showed mild anaemia in all of them. The cerebrospinal fluid showed mild protein increased in one of them $(40 \mathrm{mg} / 100 \mathrm{ml})$. The clinical manifestations of the CD patients are summarized in Table I.

The age of neuropathy presentation in patients with $\mathrm{CD}$ was $50.4 \pm 2.1$. The duration of the polyneuropathy before biopsy varied from 9 months to 21 months. All patients presented with sensory symptoms. There was severe hypoesthesia and paraesthesia in 1 and mild in 3 patients. Touch sensation was decreased in all patients. Mild distal muscle weakness was found in 3 and severe weakness in 1 patient, predominantly in the lower limbs. There were no signs of cranial neuropathy. No pronounced functional disability was found in any patient and the mean modified Rankin scale score was 3.46. Autonomic evaluation (Valsalva manoeuvre, deep breathing, tilting table and sympathetic skin-response testing) did not disclose any abnormality. There were no neurological disorders other than neuropathy.

Based on the clinical features, there was distal symmetrical sensorimotor neuropathy ( 1 of them with mild 
Table I. Clinical manifestations of patients with Crohn's disease and polyneuropathy*

\begin{tabular}{|c|c|c|c|c|c|c|c|c|}
\hline \multirow{2}{*}{ NO. OF CASE } & \multirow{2}{*}{ AGE (YEARS) } & \multirow{2}{*}{ SEX } & \multirow{2}{*}{$\begin{array}{c}\text { PATTERN OF } \\
\text { POLYNEUROPATHY }\end{array}$} & \multicolumn{5}{|c|}{ SYSTEMIC INVOLVEMENT } \\
\hline & & & & AP & DIARRHOEA & FEVER & MA & WL \\
\hline 1 & 48 & $\mathbf{M}$ & ASP & + & + & + & + & + \\
\hline 2 & 51 & $\mathrm{M}$ & SP & + & + & + & + & + \\
\hline 3 & 52 & $\mathrm{~F}$ & SP & + & + & + & + & - \\
\hline 4 & 56 & $\mathrm{M}$ & SP & + & + & + & + & + \\
\hline
\end{tabular}

asymmetries) in all cases. The electrophysiological studies were abnormal in all patients. All of them had mildly decreased motor and sensory conduction velocity with significantly reduced amplitude of the motor and sensory compound action potentials, without block or dispersion. The sural nerve action potential was absent in 1 patient. There was electromyographic evidence of chronic denervation in all patients and signs of active denervation in 1 .

\section{Histopathology}

At least three fascicles were studied in each nerve biopsy.

Epineurial and endoneurial area: There was no evidence of vasculitis. Specifically, there were no significant vessel changes or inflammatory cells infiltrate. Qualitatively, the most prominent abnormality was that of basement membrane mild thickening.

Myelinated fibres: Characteristic finding in our study is the loss of fibers in all nerve biopsies. The analysis of the histopathological findings revealed a varying degree of loss of myelinated fibers of all diameters in all cases. Mixed axonal degeneration and demyelination appears in all cases but the axonal degeneration was the predominant characteristic. There were rare presentation of onion bulb formation in all of them.

Teased fibres: Segmental demyelination was the most frequent abnormality.

Immunohistochemistry: The immunohistochemical study was negative to antihuman polyclonal antibodies $\operatorname{IgA}$, IgG, IgM, to C3 and fibrinogen.

Electron microscope: The ultrastructure study confirmed the axonal degeneration (focal accumulation of vesicles and axonal atrophy) and secondary demyelination.

Unmyelinated fibres: There was a decrease in the number of the unmyelinated fibres (morphometric study was not performed). Qualitative assessment of unmyelinated fibres demonstrated increased numbers of Schwann cell subunits devoid of axons and rare axonal sprouts suggestive of concomitant degeneration with mild regeneration.
Morphometry: Morphometric study revealed a significant loss of myelinated fibers $(2512 \pm 1137)$ (compared with a published age-matched normal control, Jacobs 1985) [20]. The mean axonal diameter was decreased $(2.77 \pm 0.68 \mu \mathrm{m})$.

The $g$ ratio was markedly decreased $(0.43 \pm 0.09)$ and supported the histopathological findings of mainly axonal involvement. The histograms of fibre diameter distributions confirmed the damage of all diameter fibers (Fig. 1).

Treatment: All patients received initial treatment with prednisone $60-80 \mathrm{mg}(1 \mathrm{mg} / \mathrm{kg}$ day). However, follow-up was not sufficiently systematic so as to permit an accurate assessment of the response to treatment.

\section{Discussion}

Extra-intestinal manifestations occur in at least $25 \%$ of $\mathrm{CD}$ patients and they can occur prior to, in conjunction with, or subsequent to active bowel disease [1-3]. Multiple other organ systems can be affected, including the bones and joints, skin, eyes, hepatobiliary system, lungs, kidneys, central and peripheral nervous system.

The exact incidence of neurological complications is unknown, with reports varying from $0.2 \%$ to $35.7 \%$ $[1,2,7,8]$. Peripheral neuropathy $(\mathrm{PN})$ ranks among the most frequent neurological complications seen in CD patients. The incidence of peripheral neuropathy varied from $0.9 \%$ to $3.6 \%[7,8]$. This difference might be explained by the systematic exclusion of all metronidazole-treated CD patients by Lossos et al. [7]. In fact, until recently peripheral neuropathy in Crohn's disease has been described, to date, only with vitamin $\mathrm{B}_{12}$ deficiency or due to oral metronidazole treatment. All forms of neuropathy in CD patients treated with metronidazole were thought to result from this medication, since CD was not considered to be a cause of PN [21]. In our study three patients were excluded and have been attributed to other causes of neuropathy (one with diabetes and vitamin $\mathrm{B}_{12}$ deficiency, one with vitamin $B_{12}$ deficiency and one with metronidazole therapy). 

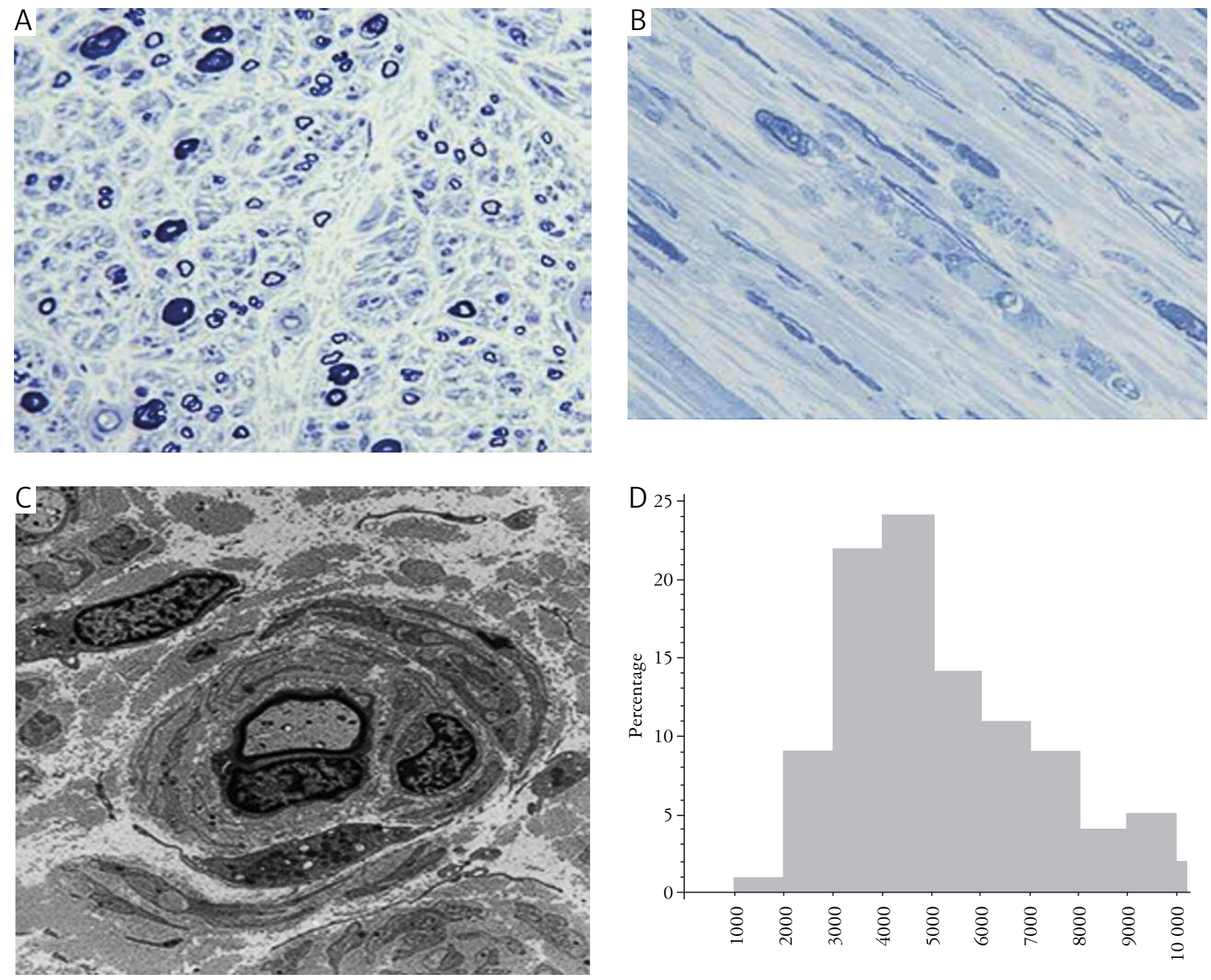

Fig. 1. Sural nerve biopsy findings: A, B - loss of myelinated nerve fibres (varying degree of loss of myelinated fibers of all diameters). Axonal degeneration. A few thinly myelinated fibers. Transverse and longitudinal sections. Toluidine blue staining. Magnification $\times 400 ; \mathrm{C}$ - onion bulb seen with electron microscopy; $\mathrm{D}$ - histogram shows unimodal fibers distribution

Although neuropathy is a frequent neurological complication in CD patients, there have been only a few studies of CD neuropathy [5-10]. The number of reports with complete clinical, laboratory, immunological, electrophysiological and nerve biopsy investigation is small $[11,12]$.

The spectrum of clinical manifestations seen in our patients was similar to that of the published reports [5-12]. Abdominal pain, diarrhoea and anaemia were the most frequent clinical presentations. The age of neuropathy presentation in our patients was $50.4 \pm 2.1$ and is similar to the study of Gondim et al. $(51.7 \pm 2.6)$.

Lossos et al. described 4 categories of neurologic involvement in $\mathrm{CD}$ : peripheral neuropathy, myopathy or myoneural junction dysfunction, cerebrovascular disease, and myelopathy [7]. Other groups of investigators have also documented demyelinating processes, seizures, encephalopathy, restless legs syndrome, sensorineural hearing loss, sacral nerve involvement and a cauda equina syndrome in patients with inflammatory bowel disease [22-28].
Neurological manifestations in our patients were peripheral polyneuropathy and there were no central nervous system findings.

As mentioned, several different PN phenotypes have been described in $\mathrm{CD}$ patients. Based on clinical and electrophysiological features, distal symmetrical sensorimotor neuropathy (1 of them with mild asymmetries) occurred in all cases in our study. Although mononeuritis multiplex is thought to be the most frequent neuropathic manifestation of $\mathrm{CD}[5,6]$, symmetrical and asymmetrical polyneuropathies $[5,6,9$, $10]$ have also been reported in a considerable proportion of patients. The high rate of symmetrical neuropathy in our patients could be possibly due to the delay between the initiation of symptoms and the clinical and neuropathological examination. At a later stage of the disease a primarily mononeuritis multiplex or asymmetric polyneuropathy could evolve towards a symmetrical picture by summation of multifocal lesions [17]. Boylu et al. reported a patient with CD and chronic inflammatory demyelinating polyneuropa- 
thy [29]. In the largest study of Gondim et al. there were three patients who met the criteria for chronic inflammatory demyelinating polyneuropathy [10]. Fuente-Fernandez et al. reported a case who fulfilled clinical and electrophysiological criteria for an acute axonal form of Guillain-Barré syndrome [30]. Moormann et al., described two patients with CD and GuillainBarré syndrome and suggested that this complication could be regarded as a possible extraintestinal manifestation of CD [9]. There was no acute evidence of polyneuropathy in our patients and nerve biopsy investigation did not reveal inflammatory cells as well as in the cerebrospinal fluid there was no protein-cell dissociation. Another feature which may be found in patients with $\mathrm{CD}$ is autonomic nerve dysfunction. Lindgren $e t$ al. investigated 33 patients with $C D$ and in spite of normal peripheral nerve function, almost half of the patients, 48\% (16/33), showed signs of autonomic neuropathy [31]. The occurrence of AN was not related to the duration or severity of $\mathrm{CD}$ or to biochemical evidence of inflammation or malabsorption of vitamins and trace elements. Ohlsson et al. reported a patient with subclinical sympathetic neuropathy which appears early in the course of $\mathrm{CD}$. In contrast to prior reports but in accordance to the study of Gondim, autonomic evaluation of our patients did not disclose any abnormality $[10,31]$.

The actual pathogenesis inciting the peripheral neuropathy in patients with $\mathrm{CD}$ is uncertain. In some cases, nutritional factors (vitamin $B_{12}$ deficiency) have been responsible and in others the peripheral neuropathy referred as a complication of medications used in treatment, such as metronidazole, infliximab (Remicade), simvastatin etc. [7, 32-35]. These conditions were excluded in our patients. Frequently, the explanation is not so clear $[36,37]$. According to Humbert et al., polyneuropathy in $\mathrm{CD}$ may have an immunological basis [9]. The efficiency of plasma exchanges in some patients suggests an autoimmune basis and vasculitis with circulating immune complexes has been identified in others $[9,11]$. The absence of any inflammatory cells and immunoglobulin deposits in the nerve biopsy of our patients cannot support but also cannot reject this hypothesis.

The analysis of the histopathological findings in our study revealed a varying degree of loss of myelinated fibers of all diameters in all cases and both axonal degeneration and demyelination were present in all cases. The dominant finding in sural nerve biopsies in the small reported studies was characterized by demyelination, axonal degeneration or both axonal degeneration and demyelination [9, 10]. Humbert et al. reported a patient with $\mathrm{CD}$ and polyneuropathy, whose nerve biopsy showed signs of regeneration, as a good prognostic feature [11]. Our study confirmed these findings, as there were signs of regeneration in all biopsies. If polyneuropathy is diagnosed early, initiation of ear- ly immunosuppressive treatment to prevent loss of nerve axons is recommended and so is the use of neuroprotective drugs [38].

Although the presence of aphthous ulcers, fissure ulcers, transmural inflammation, fistulas, lymphangiectasia, fibrous structuring and neural changes (abnormalities of the enteric nervous system are common and they have been called 'neuromatous lesions') is predominantly a feature of endoscopic mucosal biopsies, granulomas in histological sections are a key feature of $\mathrm{CD}$. Rarely does the granulomatous inflammation affect extraintestinal sites, such as the skin, liver, lungs, eyes and ovaries. The nerve biopsy findings in our patients showed no specific lesions of mucosal biopsies, as inflammatory cells or granulomas. It is unknown whether this is due to a different pathogenic mechanism or more nerve biopsy sections should be studied, as it is recommended in vascular neuropathy.

In conclusion, our study confirms that 1) CD neuropathy is a condition that should also think rule out other causes of polyneuropathy when; 2) although CD polyneuropathy is rare, it is important to recognize it in the early stage because remission depends on the immunosuppressive therapy; 3) the diagnosis of polyneuropathy is based on clinical symptoms and electrophysiological studies, but precise histology, immunohistochemistry and morphometric study of the peripheral nerve biopsy may be decisive in establishing the diagnosis; 4) the presence of regeneration is a good prognostic feature diagnosed early, initiation of early treatment to prevent loss of nerve axons is recommended; 5) as the pathophysiology of neuropathy remains unknown, these findings could prove useful in the study of $\mathrm{CD}$ polyneuropathy but further studies are needed to identify parameters likely to be helpful in the diagnosis of early nerve damage.

The authors declare no conflict of interest.

\section{References}

1. Greenstein AJ, Janowitz HD, Sachar DB. The extra-intestinal complications of Crohn's disease and ulcerative colitis: a study of 700 patients. Medicine (Baltimore) 1976; 55: 401-412.

2. Rankin GB, Watts HD, Melnyk CS, Kelley ML Jr. National Cooperative Crohn's Disease Study: extraintestinal manifestations and perianal complications. Gastroenterology 1979; 77 (4 Pt 2): 914-920.

3. Ephgrave K. Extra-intestinal manifestations of Crohn's disease. Surg Clin North Am 2007; 87: 673-680.

4. Evans PE, Pardi DS. Extraintestinal manifestations of inflammatory bowel disease: focus on the musculoskeletal, dermatologic, and ocular manifestations. Med Gen Med 2007; 9: 55.

5. Nemni R, Fazio R, Corbo M, et al. Peripheral neuropathy associated with Crohn's disease. Neurology 1987; 37: 1414-1417.

6. Chaoui F, Hellal H, Balamane M, et al. Colonic Crohn's disease complicated with peripheral neuropathy. Gastroenterol Clin Biol 1990; 14: 869-872. 
7. Lossos A, Argov Z, Ackerman Z, Abramsky O. Peripheral neuropathy and folate deficiency as the first sign of Crohn's disease. J Clin Gastroenterol 1991; 13: 442-444.

8. Elsehety A, Bertorini TE. Neurologic and neuropsychiatric complications of Crohn's disease. South Med J 1997; 90: 606-610.

9. Moormann B, Herath H, Mann O, Ferbert A. Involvement of the peripheral nervous system in Crohn disease. Nervenarzt 1999; 70: 1107-1111.

10. Gondim FA, Brannagan TH 3rd, Sander HW, et al. Peripheral neuropathy in patients with inflammatory bowel disease. Brain 2005; 128: 867-879.

11. Humbert P, Monnier G, Billerey C, et al. Polyneuropathy: an unusual extraintestinal presentation of Crohn's disease. Acta Neurol Scand 1989; 80: 301-306.

12. Larrodé P, Ramón Y Cajal S, Iñíguez C, et al. Peripheral neuropathy associated with intestinal inflammatory disease. Neurologia 2001; 16: 133-137.

13. Lichtenstein GR, Hanauer SB, Sandborn WJ; Practice Parameters Committee of American College of Gastroenterology. Management of Crohn's disease in adults. Am J Gastroenterol 2009, 104: 465-483.

14. Van Assche G, Dignass A, Reinisch W, et al.; European Crohn's and Colitis Organisation (ECCO). The second European evidencebased Consensus on the diagnosis and management of Crohn's disease: Special situations. J Crohns Colitis 2010; 4: 63-101.

15. Kissel JT, Slivka AP, Warmolts JR, Mendell JR. The clinical spectrum of necrotizing angiopathy of the peripheral nervous system. Ann Neurol 1985; 18: 251-257.

16. Kararizou E, Davaki P, Karandreas N, et al. Nonsystemic vasculitic neuropathy: a clinicopathological study of 22 cases. J Rheumatol 2005; 32: 853-858.

17. Kararizou E, Davaki P, Spengos K, et al. Churg-Strauss syndrome complicated by neuropathy: a clinicopathological study of nine cases. Clin Neuropathol 2011; 30: 11-17.

18. Dyck PJ, Giannini C, Lais A. Pathologic alterations of nerves. In: Peripheral neuropathy. Dyck PJ, Thomas PK, Griffin JW, et al. (eds.). 3rd ed. WB Saunders, Philadelphia 1993; 1424-1436.

19. Barohn RJ, Kissel JT, Warmolts JR, Mendell JR. Chronic inflammatory demyelinating polyradiculoneuropathy. Clinical characteristics, course and recommendations for diagnostic criteria. Arch Neurol 1989; 46: 878-884.

20. Jacobs JM, Love S. Qualitative and quantitative morphology of human sural nerve at different ages. Brain 1985; 108: 897-924.

21. Coxon A, Pallis CA. Metronidazole neuropathy. J Neurol Neurosurg Psychiatry 1976; 39: 403-405.

22. Brohee P, Violon P, Mavroudakis N, et al. Central nervous system lesions associated with Crohn's disease. J Neuroimaging 1997; 7: 195-198.

23. Jaussaud R, Deville JF. Central neurologic manifestations of Crohn disease. Rev Med Interne 1999; 20: 527-530.

24. de Lau LM, de Vries JM, van der Woude CJ, et al. Acute CNS white matter lesions in patients with inflammatory bowel disease. Inflamm Bowel Dis 2009; 15: 576-580.

25. Kimura K, Hunter SF, Thollander MS, et al. Concurrence of in flammatory bowel disease and multiple sclerosis. Mayo Clin Proc 2000; 75: 802-806

26. Gupta G, Gelfand JM, Lewis JD. Increased risk for demyelinating diseases in patients with inflammatory bowel disease. Gastroenterology 2005; 129: 819-826.

27. Weinstock LB, Bosworth BP, Scherl EJ, et al. Crohn's disease is associated with restless legs syndrome. Inflamm Bowel Dis 2010; 16: 275-279.

28. Demarquay JF, Caroli-Bosc FX, Buckley M, et al. Right-sided sciatalgia complicating Crohn's disease. Am J Gastroenterol 1998; 93: 2296-2298.

29. Boylu E, Toğrol E, Doğan T, Saraçoğlu M. Crohn disease and chronic inflammatory demyelinating polyneuropathy: a case report. Electromyogr Clin Neurophysiol 2010; 50: 181-185.
30. de la Fuente-Fernández R, Rubio-Nazabal E, de la IglesiaMartínez F. Guillain-Barré syndrome as an extraintestinal manifestation of Crohn's disease. Postgrad Med J 1995; 71: 437-438.

31. Lindgren S, Lilja B, Rosén I, Sundkvist G. Disturbed autonomic nerve function in patients with Crohn's disease. Scand J Gastroenterol 1991; 26: 361-366.

32. Baumgart DC, Sandborn WJ. Inflammatory bowel disease: clinical aspects and established and evolving therapies. Lancet 2007; 369: 1641-1657.

33. Contamin F, Ollat H, Lévy VG, Thierman-Duffaud D. Involvement of the peripheral and pyramidal nervous system in Crohn disease. Determining role of folic acid deficiency. Sem Hop 1983; 59: 1381-1385.

34. Duffy LF, Daum F, Fisher SE, et al. Peripheral neuropathy in Crohn's disease patients treated with metronidazole. Gastroenterology 1985; 88: 681-684.

35. Daglioglu E, Berker M, Demirci M, et al. Microscopic and electrophysiological changes on regenerating sciatic nerves of rats treated with simvastatin. Folia Neuropathol 2010; 48: 49-56.

36. Ståhlberg D, Bárány F, Einarsson K, et al. Neurophysiologic studies of patients with Crohn's disease on long-term treatment with metronidazole. Scand J Gastroenterol 1991; 26: 219-224.

37. Nemni R, Fazio R, Corbo M, et al. Peripheral neuropathy associated with Crohn's disease. Neurology 1987; 37: 1414-1417.

38. Noorafshan A, Shariat K, Karbalay-Doust S, Omidi A. Rosiglitazone protects the dorsal root ganglion cells and sciatic nerve after crush in rat. Folia Neuropathol 2011; 49: 142-151.

\section{Address for correspondence}

Evangelia Kararizou, MD, Assistant Professor

Section of Neuropathology

Neurological Clinic, Aeginition Hospital

72-74 Vas.Sofias av., 11528,

Athens, Greece

tel. 210-7289282

fax 210-7216474

e-mail: ekarariz@med.uoa.gr 